\title{
Métodos estatísticos para validade e reprodutibilidade em pesquisas na área de Radiologia Odontológica
}

\author{
Anne C. A. Ramos (IC), Thiago O. Gamba (PG), Karine L. Cortellazzi (PQ), Gláucia M. B. Ambrosano \\ (PQ)
}

\section{Resumo}

O objetivo do presente estudo foi definir quais os métodos estatísticos mais adequados para avaliação da validade e reprodutibilidade em pesquisas da área de Radiologia Odontológica. Foram analisados artigos científicos da área, bancos de dados de pesquisas prévias realizadas e simuladas novas situações considerando diferentes tamanhos de amostra, amplitude e diferentes prevalências. Observou-se que a indicação do teste estatístico a ser utilizado depende do tipo de variável estudada e da pergunta da pesquisa. Em estudos onde será utilizado o teste Kappa a prevalência da amostra deve ser próxima a 50\%.

Palavras Chave: Reprodutibilidade dos Testes, Análise Estatística, Tomografia Computadorizada de Feixe Cônico.

\section{Introdução}

Para o correto diagnóstico, a Radiologia Odontológica necessita de aprimoramentos, que são realizados por meio das pesquisas científicas. A Estatística é uma ferramenta valiosa nas análises dos dados das pesquisas cientificas em diagnósticos e está ao alcance de todos. É muito importante se conhecer os testes utilizados e suas eficácias, que podem dar a garantia de um resultado mais preciso, ou uma conclusão errônea dos resultados da pesquisa ('Larheim et al. 1996). Frente a esses conceitos, o objetivo do presente estudo foi definir quais os métodos estatísticos mais adequados para avaliação da validade e reprodutibilidade em pesquisas da área de Radiologia Odontológica.

\section{Resultados e Discussão}

Foram realizadas simulações a partir de dados coletados de pesquisas em Radiologia Odontológica, variando-se o tamanho da amostra e a prevalência do evento no caso do teste Kappa e o tamanho da amostra e a amplitude dos dados no caso da análise de correlação intra classe. A prevalência do evento estudado é muito importante no cálculo da estatística kappa, sendo que 0 pesquisador deve se preocupar em trabalhar com prevalência em torno de $50 \%$ na amostra. O tamanho da amostra é muito importante para a precisão, ou seja, quanto maior o tamanho da amostra menor é a amplitude do intervalo de confiança, e mais confiável é a estimativa do kappa. Quanto ao coeficiente de correlação intraclasse, pode-se observar que a amplitude e o tamanho da amostra influenciam nos resultados e que outros estudos devem ser realizados para orientar os pesquisadores no planejamento de estudos que utilizarão essa análise.
Tabela 1. Distribuição de frequências dos testes utilizados.

\begin{tabular}{ccc}
\hline Teste & Frequência & Porcentagem \\
\hline Kappa & 16 & $50 \%$ \\
Kappa P. & 7 & $21,8 \%$ \\
CCl & 7 & $21,8 \%$ \\
Sensibilidade & 13 & $40,6 \%$ \\
Especificidade & 13 & $40,6 \%$ \\
VPP & 6 & $18,7 \%$ \\
VPN & 6 & $18,7 \%$ \\
Curva ROC & 9 & $28,1 \%$ \\
\hline
\end{tabular}

${ }^{*} \mathrm{CCl}$ : coeficiente de correlação intra classe. VPP: valor preditivo positivo. VPN: valor preditivo negativo.

\section{Conclusões}

Pode-se concluir que, a indicação do teste a ser utilizado depende do tipo de variável analisada no estudo e da pergunta da pesquisa, a amplitude do intervalo de confiança, ou seja, a precisão da estimativa depende do tamanho da amostra. Em estudos de reprodutividade em que será utilizado o teste Kappa a prevalência do evento estudado na amostra deve estar próximo a $50 \%$. A amplitude dos dados influencia na análise do coeficiente de correlação intra classe

\section{Agradecimentos}

Agradecemos ao Programa Institucional de Bolsas de Iniciação Científica (PIBIC) pelo apoio durante a realização deste projeto.

\footnotetext{
Larheim, T. A.; Svanaes, D. B. Am J Orthod Dentofacial Orthop. 1996, 90(1):45-51.
} 